\title{
Hindbrain Noradrenergic Lesions Attenuate Anorexia and Alter Central cFos Expression in Rats after Gastric Viscerosensory Stimulation
}

\author{
Linda Rinaman \\ Department of Neuroscience, University of Pittsburgh, Pittsburgh, Pennsylvania 15260
}

\begin{abstract}
Behavioral, autonomic, and endocrine outputs of the CNS are subject to important feedback modulation by viscerosensory signals that are conveyed initially to the hindbrain nucleus of the solitary tract (NST). In the present study, noradrenergic (NA) neurons [i.e., those that express the NA synthetic enzyme dopamine $\beta$ hydroxylase $(\mathrm{DbH})]$ in the caudal NST were lesioned to determine their role in mediating anorexic responses to gastric stimulation and in conveying gastric sensory signals to the hypothalamus and amygdala. For this purpose, saporin toxin conjugated to an antibody against $\mathrm{DbH}$ was microinjected bilaterally into the caudal NST in adult rats. Control rats received similar microinjections of vehicle. Several weeks later, rats were tested for the ability of systemic cholecystokinin octapeptide (CCK) $(0$ or $10 \mu \mathrm{g} / \mathrm{kg})$ to inhibit food intake. CCK-induced anorexia was significantly attenuated in toxin-treated rats. Rats subsequently were used in a terminal cFos study to determine central neural activation patterns after systemic CCK or vehicle and to evaluate lesion extent. Toxin-induced loss of DbH-positive NST neurons was positively correlated with loss of CCK-induced anorexia. Hypothalamic cFos expression was markedly attenuated in lesioned rats after CCK treatment, whereas CCK-induced neural activation in the parabrachial nucleus and amygdala appeared normal. These findings suggest that hindbrain NA neurons are an integral component of brainstem circuits that mediate CCK-induced anorexia and also are necessary for hypothalamic but not parabrachial or amygdala responses to gastric sensory stimulation.
\end{abstract}

Key words: gastrointestinal; vagus; nucleus of the solitary tract; hypothalamus; amygdala; parabrachial nucleus; saporin toxin

\section{Introduction}

Behavioral, autonomic, and endocrine outputs of hypothalamic and limbic forebrain circuits are subject to important feedback modulation by viscerosensory signals from the gut, and bidirectional interactions between gastrointestinal function and behavioral-cognitive state are well recognized. These interactions contribute to the clinical problem of anorexia after chemotherapeutic cancer treatment and may underlie the high comorbidity of gastrointestinal disorders with anxiety, depression, and other psychiatric disorders (Ballenger et al., 2001; Mayer et al., 2001). However, the neurochemistry and functional organization of central pathways between the gut, hypothalamus, and limbic forebrain are incompletely understood.

Systemic administration of cholecystokinin octapeptide (CCK) provides a straightforward way to activate central gastric viscerosensory pathways to study their functional organization. CCK binds to receptors on gastric vagal afferents, thereby stimulating viscerosensory inputs to the medullary nucleus of the solitary tract (NST) (Raybould et al., 1985; Day et al., 1994; Schwartz and Moran, 1998). Small doses of CCK in rats and in humans

\footnotetext{
Received May 22, 2003; revised Sept. 10, 2003; accepted Sept. 11, 2003.

This work was supported by National Institutes of Health Grant MH 59911. I thank Carl Wonders, Heather Wilson and Victoria Maldovan for their valuable technical contributions to this work.

Correspondence should be addressed to Dr. Linda Rinaman, University of Pittsburgh, 446 Crawford Hall, Pittsburgh, PA 15260. E-mail: rinaman@pitt.edu.

Copyright $\odot 2003$ Society for Neuroscience $\quad$ 0270-6474/03/2310084-09\$15.00/0
}

mimic the physiological satiety effects of endogenous CCK released from the gut postprandially (Schwartz and Moran, 1998). Conversely, large doses of CCK (i.e., $10-100 \mu \mathrm{g} / \mathrm{kg}$ ) produce anorexia that generally is accompanied by nausea, pituitary release of stress hormones, and, in humans, anxiety (Verbalis et al., 1986a,b; Kamilaris et al., 1992; McCutcheon et al., 1992; Greenough et al., 1998). Combined radiofrequency lesions of the area postrema (AP) and portions of the medial NST in rats virtually eliminate the anorexigenic effect of low doses of exogenous CCK (i.e., 1-2 $\mu \mathrm{g} / \mathrm{kg}$ ) (Edwards et al., 1986). However, these lesions spared a portion of the caudal medial and commissural NST, and a higher dose of CCK (i.e., $8 \mu \mathrm{g} / \mathrm{kg}$ ) was still effective in inhibiting food intake (Edwards et al., 1986).

In rats, systemic CCK activates ascending projections to the hypothalamus and amygdala that arise primarily from noradrenergic (NA) [i.e., dopamine $\beta$ hydroxylase ( $\mathrm{DbH}$ ) positive] neurons of the A2 cell group, located within the caudal medial and commissural NST (Rinaman et al., 1995; Myers and Rinaman, 2002). CCK also activates a smaller contingent of noncatecholaminergic NST neurons with ascending projections; these are immunoreactive for glucagon-like peptide 1 (GLP-1) (Rinaman, 1999; Myers and Rinaman, 2002). Other ascending viscerosensory pathways activated by CCK include NST projections that are relayed through the ventrolateral medulla (VLM) and pontine parabrachial nucleus $(\mathrm{PBN})$. Ascending NA pathways are implicated in viscerosensory activation of the hypothalamus 


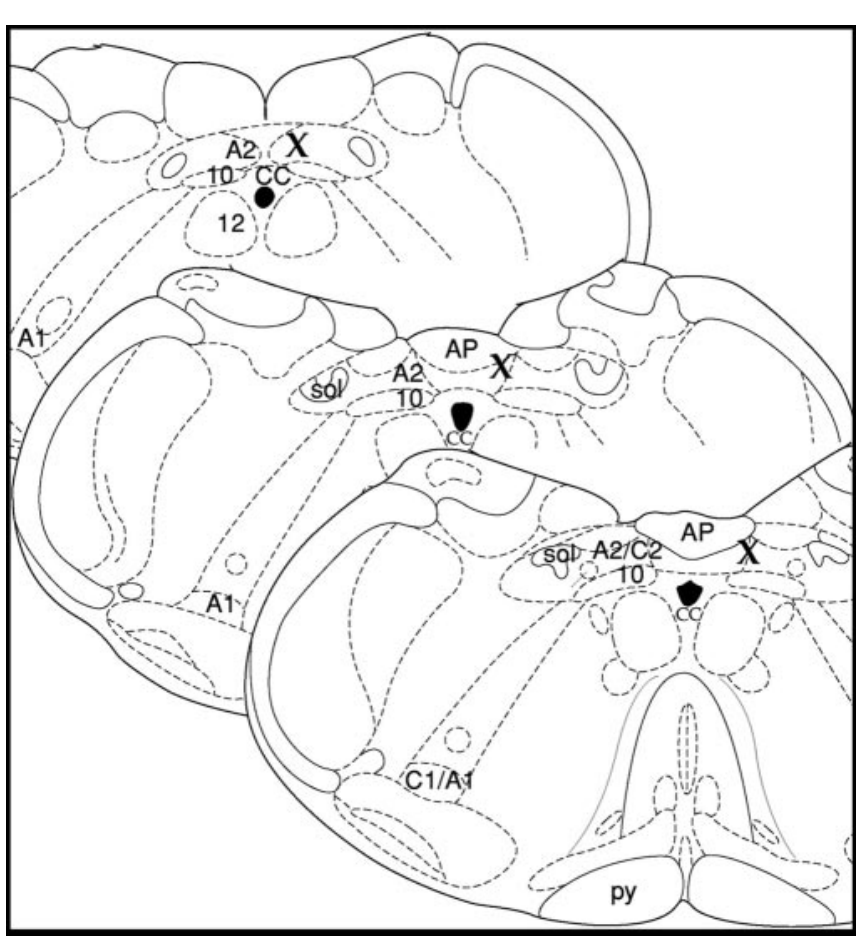

Figure 1. Schematic representation of NST sites $(X)$ targeted for $50 \mathrm{nl}$ microinjections of toxin or vehicle. Similar contralateral sites were targeted in each rat. Schematics adapted from commercially available digital files (Paxinos and Watson, 1998). CC, Central canal; py, pyramidal tract; sol, solitary tract.

(Sawchenko and Swanson, 1981; Ueta et al., 1993; Sawchenko et al., 2000), but the relative contributions of NA and noncatecholaminergic projections from the NST to the hypothalamus and other central viscerosensory circuit components are unclear. A novel immunotoxin comprising an antibody to the NA synthetic enzyme $\mathrm{DbH}$ conjugated to saporin toxin has been used successfully to selectively destroy hindbrain neurons that contain DbH (Wrenn et al., 1996; Madden et al., 1999; Ritter et al., 2001; Fraley et al., 2002; Fraley and Ritter, 2003). The present study used this toxin to selectively destroy $\mathrm{DbH}$-positive neurons in the caudal NST and thereby determine their role in mediating the anorexigenic and CNS activation effects of exogenous CCK.

\section{Materials and Methods}

Animals. All procedures conformed to National Institutes of Health guidelines and were approved by the University of Pittsburgh Animal Care and Use Committee. Data from 20 adult male Sprague Dawley rats (Harlan Sprague Dawley, Indianapolis, IN) are included in this report. Rats were singly housed with ad libitum access to water and pelleted rat chow (Purina, St. Louis, MO) except during food intake experiments. Colony rooms were maintained at $22-23^{\circ} \mathrm{C}$ and kept on a $12 \mathrm{hr}$ light/dark cycle, with lights on from 7:00 A.M. to 7:00 P.M. Rats weighed 180-225 $\mathrm{gm}$ at the time of toxin injection surgery and 200-325 gm at the time of food intake experiments and the terminal cFos study.

Experimental groups. Rats received bilateral injections of anti-DbHsaporin toxin (toxin; $n=10$; Advanced Targeting Systems, San Diego, CA) or $0.15 \mathrm{M} \mathrm{NaCl}$ (vehicle; $n=10$ ) into the caudal NST. Each rat was used in a food intake experiment and in a terminal cFos study.

NST microinjection. Toxin or vehicle microinjections were made into the NST at six sites (Fig. 1). Injection sites encompassed the NST region that contains the highest incidence of cFos expression by $\mathrm{DbH}$-positive neurons after CCK treatment, corresponding to the location of the A2 cell group within the caudal medial NST (Sawchenko and Swanson, 1982; Rinaman et al., 1993; Rinaman et al., 1995). Rats were anesthetized with halothane (2-5\% in $100 \%$ oxygen) and secured in a stereotaxic

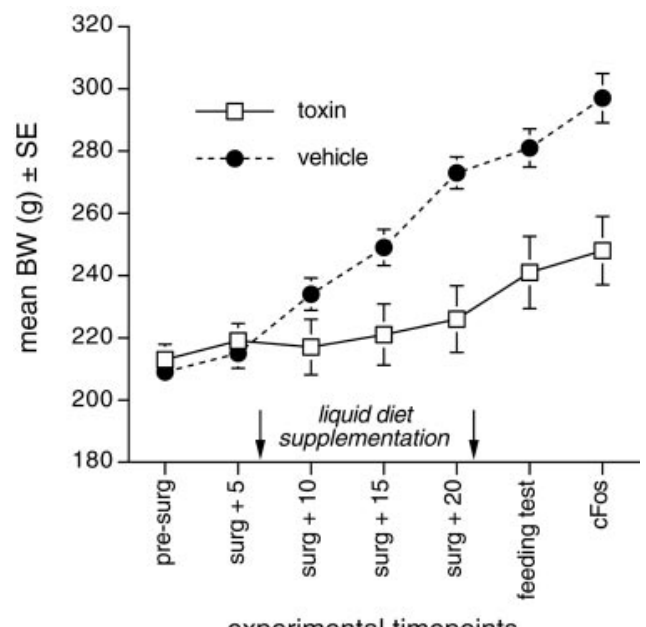

experimental timepoints

Figure 2. Line graph plotting the average body weights of rats at several experimental time points after bilateral injections of saporin toxin or vehicle into the caudal NST. Toxin-injected rats weighed $\sim 14-17 \%$ less than vehicle-injected controls at the time of feeding tests and terminal cFos studies, respectively. The 2 week period of Ensure liquid diet supplementation for both groups is indicated by arrows.

frame using blunt ear bars, with the head ventroflexed. The skin over the dorsal neck surface was shaved and incised, and the neck muscles were retracted and bluntly dissected to expose the meninges overlying the dorsal surface of the caudal medulla. With the aid of a surgical microscope, the meninges were cut with a sterile needle to reveal the AP. Under visual guidance, a glass micropipette tip (outer diameter of 50-75 $\mu \mathrm{m}$ ) filled with toxin or vehicle and affixed to a $0.5 \mu$ l Hamilton syringe was positioned on the midline at the caudal limit of the AP and was then moved $0.25 \mathrm{~mm}$ lateral and $0.5 \mathrm{~mm}$ below the medullary surface for the first, most caudal injection site. The second injection site was located 0.25 mm below the lateral border of the AP at its midrostrocaudal extent, and the third site was located $0.25 \mathrm{~mm}$ below the lateral border of the AP at its most rostral extent (Fig. 1). The three NST injection sites were then duplicated contralaterally. At each injection site, $50 \mathrm{nl}$ of sterile $0.15 \mathrm{M}$ $\mathrm{NaCl}$ vehicle containing 0 or $5 \mathrm{ng}$ of toxin was delivered by manual pressure injection over $\sim 30 \mathrm{sec}$. Toxin was freshly prepared from a frozen stock solution within $2 \mathrm{hr}$ of injection. Results from a published report (Madden et al., 1999) and additional pilot studies confirmed that this concentration and volume of toxin destroyed the majority of $\mathrm{DbH}$ positive neurons within the caudal NST (see Results). Lower toxin doses were less effective, whereas higher doses produced marked tissue necrosis. After each microinjection, the pipette tip was left in place for 1-2 min and then withdrawn. The skin incision was closed with stainless steel clips after the final injection. Rats were returned to their home cages after recovery from anesthesia.

The fact that saporin toxin microinjected near NA cell bodies effectively lesions those cells provides empirical evidence for vesicular release and concomitant extracellular exposure of $\mathrm{DbH}$ within the toxin injection site (Madden et al., 1999). The dorsal vagal complex (DVC) receives relatively sparse input from other medullary and pontine NA cell groups but is richly innervated by $\mathrm{DbH}$-positive nerve terminals of presumably local origin. Somatic and/or dendritic vesicular release from NA neurons also may occur, as has been reported for local monoamine release within the locus ceruleus and other central sites (Pudovkina et al., 2001).

Postsurgical maintenance. Rats with NST vehicle injections attained their presurgical body weights (BWs) within 2-3 d after surgery and continued to gain BW thereafter (Fig. 2). Conversely, although the 10 rats with bilateral toxin injections also attained their presurgical BWs within 2-3 d, several began to lose BW beginning 6-7 d after surgery. This effect is consistent with the delayed time course of toxin-induced neuronal death (Madden et al., 1999). To encourage food intake in these rats while maintaining consistent dietary treatment within both groups, all vehicleand toxin-injected rats were given daily overnight access to $25 \mathrm{ml}$ of a 
palatable liquid diet (vanilla-flavored Ensure) in addition to ad libitum chow, regardless of their postsurgical BW profile. Liquid diet access was initiated $7 \mathrm{~d}$ after NST injection surgery and discontinued after 2 weeks, by which time the majority were consuming their full allotment of liquid diet plus additional chow each day, and all had surpassed their presurgical BWs. Rats continued to gain at least $1 \mathrm{gm}$ of BW per day after termination of liquid diet access. Three toxin-injected rats did not consume adequate amounts of chow or liquid diet to maintain BW. These rats were killed without data collection and are not included in the reported $n$ values.

Food intake experiment. At least $10 \mathrm{~d}$ before the terminal cFos experiment described below, rats with bilateral NST injections of toxin $(n=10)$ or vehicle $(n=10)$ were used to determine the effects on food intake produced by intraperitoneal administration of CCK. For this purpose, rats were moved from their original colony room to a new environment with the same $12 \mathrm{hr}$ light/dark cycle. Rats were housed individually in clear Plexiglas boxes $(25 \times 30 \mathrm{~cm}$ floor, $22 \mathrm{~cm}$ height $)$ with stainless steel rod floors, each equipped with a computer-driven pellet delivery and monitoring system (Med Associates, E. Fairfield, VT). Drinking water was available ad libitum from sipper tubes within each box. Food access was restricted to a daily $4 \mathrm{hr}$ period beginning at lights out (7:00 P.M.), when a single $45 \mathrm{mg}$ chow pellet (Precision Dustless Pellets; Bio-Serv; Frenchtown, NJ) was delivered automatically to a shallow feeding trough in each cage, thereby breaking a photobeam crossing the base of the trough. A new pellet was delivered automatically each time the preceding pellet was removed. The cumulative number of pellet deliveries (i.e., photobeam breaks) was recorded automatically at 15 min intervals during the $4 \mathrm{hr}$ feeding period. Cage trays beneath the open rod floors were routinely inspected at the end of the feeding period to ensure that delivered pellets were being consumed. Data were collected and stored using Med PC software (Med Associates). Rats were acclimated to the new environment and feeding schedule for 1 week before testing, by which time stable daily $4 \mathrm{hr}$ food intakes were achieved. Rats also were acclimated to gentle handling and occasional intraperitoneal saline injections during this period.

On testing day 1 , all rats were injected intraperitoneally with $2.0 \mathrm{ml}$ of $0.15 \mathrm{M} \mathrm{NaCl}$ vehicle. Food access was initiated within $2 \mathrm{~min}$, at lights out (7:00 P.M.). Cumulative pellet delivery data were collected for $4 \mathrm{hr}$. On testing day 2, all rats were injected intraperitoneally with $2.0 \mathrm{ml}$ of vehicle containing CCK (sulfated octapeptide; $10 \mu \mathrm{g} / \mathrm{kg}$ BW; Sigma, St. Louis, $\mathrm{MO}$ ). Food access was initiated within $2 \mathrm{~min}$ after CCK injection, at lights out (7:00 P.M.). Cumulative pellet delivery data were collected for $4 \mathrm{hr}$. Thus, each rat served as its own control for the effects of intraperitoneal vehicle vs. intraperitoneal CCK treatment on subsequent $4 \mathrm{hr}$ food intake. Rats were kept in the same housing environment but were returned to ad libitum chow access after testing day 2.

Food intake data analysis. BWs ranged from 200 to $293 \mathrm{gm}$ on testing days; thus, intake (i.e., pellet delivery) data for each rat were converted to grams (number of pellets $\times 45 \mathrm{mg}$ ) and expressed as percentage BW. Intake data were combined $a$ priori according to NST injection group (i.e., toxin vs vehicle) and expressed as group mean \pm SE. Treatmentrelated differences in food intake were tested for statistical significance by using ANOVA, with NST injection plus intraperitoneal treatment (i.e., NST toxin or NST vehicle plus intraperitoneal CCK or intraperitoneal vehicle) as the independent variable. When $F$ values indicated a significant main effect of treatment on food intake, the ANOVA was followed up with post hoc $t$ tests using Dunn's (Bonferonni) correction for multiple comparisons. Differences were considered significant when $p<0.05$. Feeding data in rats with NST toxin injections also were analyzed by correlating CCK-induced feeding suppression with toxin-induced loss of $\mathrm{DbH}$-positive neurons (see Results).

CCK-induced cFos experiment. A terminal cFos study was performed in each rat at least 4 weeks after NST injection surgery and at least $3 \mathrm{~d}$ after the food intake experiment. For this purpose, five rats from each NST injection group were injected intraperitoneally with $2.0 \mathrm{ml}$ of $0.15 \mathrm{M}$ $\mathrm{NaCl}$ vehicle, and five rats from each group were injected with vehicle containing CCK (10 $\mu \mathrm{g} / \mathrm{kg}$; Sigma). Injections were made between 9:00 A.M. and 10:00 A.M.

Rats were returned to their home cages immediately after intraperito- neal injection and left undisturbed for 60-75 min. Rats then were anesthetized with sodium pentobarbital (Nembutal; $50 \mathrm{mg} / \mathrm{kg}$, i.p.) and perfused through the heart with $0.9 \% \mathrm{NaCl}$, followed by fixative solution (McLean and Nakane, 1974) containing 4\% paraformaldehyde, $1.4 \%$ lysine, and $0.2 \%$ sodium metaperiodate in $0.1 \mathrm{~m}$ sodium phosphate (buffer). Fixed brains were removed from the skull, postfixed overnight, and then cryoprotected for $24-48 \mathrm{hr}$ in $20 \%$ sucrose. A freezing stage microtome was used to cut coronal sections with a thickness of $30 \mu \mathrm{m}$ from the caudal extent of the DVC through the rostral extent of the corpus callosum. Sections were collected in six serially adjacent sets and stored at $-20^{\circ} \mathrm{C}$ in cryopreservant solution (Watson et al., 1986).

Immunocytochemistry. Primary and secondary antisera were diluted in buffer containing $0.3 \%$ Triton X-100 and $1 \%$ normal donkey serum. Dual-immunoperoxidase localization of $\mathrm{DbH}$ and $\mathrm{cFos}$ was performed to simultaneously assess the extent of NST lesions and determine treatment-induced neuronal activation. For this purpose, one of the six sets of tissue sections from each rat was incubated for $48 \mathrm{hr}$ at $4^{\circ} \mathrm{C}$ in rabbit anti-cFos (1:50,000; provided by Dr. P. Larsen, Centre of Clinical and Basic Research, Laboratory of Obesity Research, Ballerup, Denmark). The specificity of this antibody for cFos protein has been reported previously (Rinaman et al., 1997). Sections were rinsed and processed for blue-black immunoperoxidase localization of nuclear Fos labeling using biotinylated donkey anti-rabbit IgG (1:500; Jackson ImmunoResearch, West Grove, PA), Elite Vectastain avidin-biotin reagents (Vector Laboratories, Burlingame, $\mathrm{CA}$ ), and a nickel-enhanced DAB reaction. After cFos immunostaining, sections were incubated for $48 \mathrm{hr}$ at $4^{\circ} \mathrm{C}$ in mouse anti- $\mathrm{DbH}(1: 30,000$; Chemicon, Temecula, CA) and processed for brown immunoperoxidase localization of cytoplasmic $\mathrm{DbH}$ using biotinylated donkey anti-mouse $\operatorname{IgG}(1: 500)$, Elite Vectastain reagents, and a nonenhanced $\mathrm{DAB}$ reaction. Additional sets of tissue sections from each rat were processed similarly for dual-immunoperoxidase localization of cFos and calcitonin gene-related peptide (CGRP) (1:20,000; Peninsula Laboratories, Belmont, CA) or cFos and oxytocin (OT) (1:20,000; Peninsula Laboratories). Immunoreacted sections were mounted out of sodium phosphate buffer onto charged glass microscope slides (SuperFrost Plus; Fisher Scientific, Pittsburgh, PA), dehydrated in a graded ethanol series, defatted in xylene, and coverslipped with Histomount (VWR Scientific, West Chester, PA).

In a selected subset of rats receiving bilateral NST toxin injections and subsequent CCK treatment before being killed $(n=3)$, additional sets of tissue sections were processed as described above for dualimmunoperoxidase localization of cFos plus tyrosine hydroxylase $(\mathrm{TH})$ (1:50,000; monoclonal antibody; Chemicon) or cFos plus GLP-1 (1:10,000; rabbit polyclonal antibody; Peninsula Laboratories). Immunostaining patterns were used to determine whether NST toxin injections destroyed dopaminergic (i.e., TH-positive but $\mathrm{DbH}$-negative) neurons in the NST and vagal motor nucleus or GLP-1-positive neurons within the NST.

Quantitative analysis of immunolabeling. Cell counting was performed to document NST lesion extent and treatment-induced cFos expression in each animal. Counts of DbH-positive neurons in the NST and VLM were performed bilaterally in every sixth section (180 $\mu \mathrm{m}$ frequency) through the rostrocaudal extent of the $\mathrm{AP}$ (four to six tissue sections per rat; five rats per group). GLP-1 neurons within the caudal NST also were counted bilaterally in three toxin-injected rats with the most extensive loss of DbH-positive neurons (see Results). Cells were counted using a $20 \times$ microscope objective on a Zeiss (Oberkochen, Germany) Axioplan 2 microscope. All DbH- and GLP-1-immunopositive profiles that were clearly perikaryal (rather than dendritic, axonal, or ambiguous) were counted, regardless of whether their nucleus was visible in the section.

Overall treatment-induced NST neural activation was determined by counting cFos-immunopositive profiles within the NST bilaterally in tissue sections through the rostrocaudal extent of the AP. The proportion of DbH-positive VLM cells that were colabeled for nuclear cFos was determined. Cells were considered cFos positive if their nucleus contained detectable blue-black immunolabeling, regardless of labeling intensity and cFos negative if they displayed no visible nucleus or a nucleus lacking cFos immunolabeling.

An effort was made to standardize the more rostral brainstem and forebrain regions subjected to quantitative analysis of treatment-induced 
Table 1. Individual and group mean cell counts (average per tissue section)

\begin{tabular}{|c|c|c|c|c|c|c|c|c|}
\hline Rat & $\begin{array}{l}\text { NST } \\
\text { injection }\end{array}$ & $\begin{array}{l}\text { Intrapentoneal } \\
\text { treatment }\end{array}$ & $\begin{array}{l}\text { NST } \\
\text { \#DbH }\end{array}$ & $\begin{array}{l}\text { NST } \\
\text { \#CFoS }\end{array}$ & $\begin{array}{l}\text { VLM } \\
\text { \#DbH; cFos\% }\end{array}$ & $\begin{array}{l}\text { Lateral PBN } \\
\# C G R P+C F o s\end{array}$ & $\begin{array}{l}\text { PVN } \\
\# 0 \mathrm{O}+\mathrm{CFos}\end{array}$ & $\begin{array}{l}\text { CeA } \\
\text { \#CFos }\end{array}$ \\
\hline 1 & Toxin & CCK & 3.2 & 172.6 & $21.5 ; 4.7 \%$ & 38.0 & 5.3 & 297.4 \\
\hline 2 & Toxin & CCK & 11.3 & 157.7 & $19.2 ; 5.2 \%$ & 42.4 & 11.3 & 206.3 \\
\hline 3 & Toxin & CCK & 34.8 & 185.3 & $28.9 ; 14.3 \%$ & 26.5 & 6.0 & 192.6 \\
\hline 4 & Toxin & CCK & 39.6 & 194.1 & $30.0 ; 10.1 \%$ & 41.8 & 4.3 & 267.8 \\
\hline \multirow[t]{2}{*}{5} & Toxin & CCK & 41.4 & 218.4 & $24.4 ; 20.8 \%$ & 30.3 & 2.6 & 126.5 \\
\hline & \multicolumn{2}{|c|}{ Group mean \pm SE } & $26.1 \pm 7.9$ & $185.6 \pm 10.2$ & $24.8 \pm 3.1 ; 11.0 \pm 3.1 \%$ & $35.8 \pm 3.2$ & $5.9 \pm 1.5$ & $218.1 \pm 30.0$ \\
\hline 6 & Toxin & Vehicle & 0.3 & 39.1 & $21.9 ; 5.4 \%$ & 7.5 & 9.5 & 16.3 \\
\hline 7 & Toxin & Vehicle & 1.4 & 12.3 & $15.7 ; 13.3 \%$ & 4.5 & 7.3 & 27.8 \\
\hline 8 & Toxin & Vehicle & 19.2 & 15.4 & $22.2 ; 4.5 \%$ & 19.3 & 5.6 & 21.2 \\
\hline 9 & Toxin & Vehicle & 21.5 & 22.4 & $23.0 ; 0 \%$ & 11.3 & 14.2 & 11.3 \\
\hline \multirow[t]{2}{*}{10} & Toxin & Vehicle & 52.5 & 11.3 & $30.1 ; 3.3 \%$ & 2.3 & 4.3 & 4.4 \\
\hline & \multicolumn{2}{|c|}{ Group mean \pm SE } & $19.0 \pm 9.5$ & $20.1 \pm 5.1$ & $22.6 \pm 2.3 ; 5.3 \pm 2.2 \%$ & $8.9 \pm 3.0$ & $8.2 \pm 1.7$ & $16.2 \pm 4.0$ \\
\hline 11 & Vehicle & CCK & 82.7 & 160.6 & $35.7 / 24.7 \%$ & 41.5 & 27.5 & 221.9 \\
\hline 12 & Vehicle & CCK & 95.7 & 174.0 & $36.5 / 19.4 \%$ & 37.5 & 29.3 & 142.5 \\
\hline 13 & Vehicle & CCK & 108.3 & 221.3 & $30.8 / 20.5 \%$ & 31.3 & 48.6 & 87.8 \\
\hline 14 & Vehicle & CCK & 115.0 & 205.1 & $43.6 / 28.0 \%$ & 48.3 & 42.4 & 185.3 \\
\hline \multirow[t]{2}{*}{15} & Vehicle & CCK & 120.0 & 263.4 & $31.4 / 31.3 \%$ & 59.6 & 36.5 & 204.1 \\
\hline & \multicolumn{2}{|c|}{ Group mean \pm SE } & $104.3 \pm 6.8$ & $204.9 \pm 18.2$ & $35.6 \pm 2.3 ; 24.8 \pm 2.8 \%$ & $43.6 \pm 4.9$ & $36.9 \pm 4.0$ & $168.3 \pm 24.1$ \\
\hline 16 & Vehicle & Vehicle & 90.0 & 21.0 & $32.6 / 0 \%$ & 12.5 & 4.3 & 8.2 \\
\hline 17 & Vehicle & Vehicle & 107.2 & 9.2 & $29.6 / 4.6 \%$ & 5.3 & 6.4 & 13.6 \\
\hline 18 & Vehicle & Vehicle & 105.3 & 9.6 & $28.4 / 9.4 \%$ & 14.6 & 13.5 & 29.7 \\
\hline 19 & Vehicle & Vehicle & 115.8 & 18.6 & $30.5 / 8.8 \%$ & 9.5 & 6.3 & 24.3 \\
\hline \multirow[t]{2}{*}{20} & Vehicle & Vehicle & 124.3 & 3.4 & $32.5 / 6.1 \%$ & 5.3 & 9.1 & 17.0 \\
\hline & \multicolumn{2}{|c|}{ Group mean \pm SE } & $108.5 \pm 5.7$ & $12.4 \pm 3.3$ & $30.7 \pm 0.8 ; 5.8 \pm 1.7 \%$ & $9.4 \pm 1.9$ & $7.9 \pm 1.6$ & $18.6 \pm 3.8$ \\
\hline
\end{tabular}

cFos expression. Thus, cFos labeling in the pontine PBN was quantified by determining the number of CGRP-positive neurons in the lateral PBN that were activated to express cFos, using counting criteria similar to those described above. Tissue sections were examined bilaterally through the rostrocaudal extent of cellular CGRP labeling in the lateral PBN (three to four sections spaced by $180 \mu \mathrm{m}$ per rat). Activation in the hypothalamic paraventricular nucleus (PVN) was quantified by determining the number of magnocellular and parvocellular OT-positive neurons that contained nuclear $\mathrm{cFos}$ labeling. In each rat, two tissue sections through the PVN (spaced by $180 \mu \mathrm{m}$ ) containing both medial parvocellular and lateral magnocellular PVN subnuclei were selected for analysis. Amygdala activation was determined using CGRP fiber immunolabeling to distinguish the cytoarchitectural boundaries of the central nucleus of the amygdala (CeA). All cFos-positive profiles within those CeA boundaries were counted in tissue sections through the rostrocaudal extent of fibrous CGRP labeling (five to seven sections spaced by $180 \mu \mathrm{m}$ in each rat).

Regional cell count data for each rat are expressed as the number of cells counted in each region bilaterally, divided by the number of analyzed tissue sections through the region (Table 1). Treatment group data are expressed as mean \pm SE. Between-group differences in count values for each region were tested for statistical significance by using ANOVA, with treatment condition (i.e., NST toxin or NST vehicle plus intraperitoneal CCK or intraperitoneal vehicle) as the independent variable. When $F$ values indicated a significant main effect of treatment on cell count values, the ANOVA was followed up with post hoc $t$ tests using Dunn's (Bonferonni) correction for multiple comparisons. Differences were considered significant when $p<0.05$.

Photography and preparation of illustrations. Tissue sections were photographed using Kodak Ektachrome 160T color slide film (Eastman Kodak, Rochester, NY). A Polaroid SprintScan 35 slide scanner was used to import photographic images into Adobe Photoshop 5.0 (Adobe Systems, San Jose, CA).

\section{Results}

The results of this study support the view that NA neurons in the caudal NST are a necessary component of central circuits that

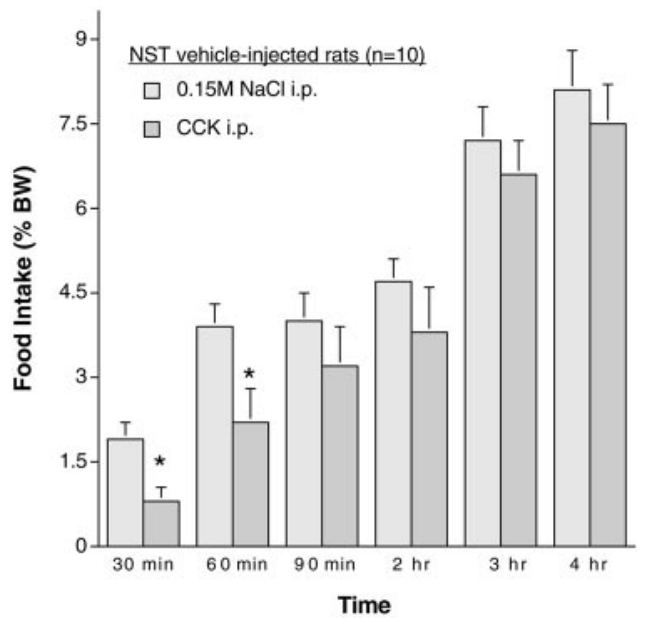

Figure 3. Food intake in rats with previous NST vehicle injections $(n=10)$ after control treatment $(0.15 \mathrm{~m} \mathrm{NaCl})$ and after CCK $(10 \mu \mathrm{g} / \mathrm{kg})$. CCK treatment significantly inhibited food intake at the 30 and 60 min time points $\left({ }^{*} p<0.05\right.$ compared with intake after control treatment).

underlie inhibition of food intake after CCK treatment. NA neurons also appear to be necessary for conveying CCK-related gastric sensory signals to the PVN but appear unnecessary for conveying such signals to the lateral $\mathrm{PBN}$ and $\mathrm{CeA}$.

\section{NST injection effects on CCK-induced anorexia}

Food intake after intraperitoneal vehicle $(0.15 \mathrm{M} \mathrm{NaCl})$ treatment was similar in rats with previous NST injections of vehicle (Fig. 3) or toxin (Fig. 4). As expected, CCK treatment suppressed food intake in rats with NST vehicle injections (Fig. 3). Analysis of data from individual rats confirmed the generality of this effect; that is, CCK significantly inhibited food intake in every rat within the 


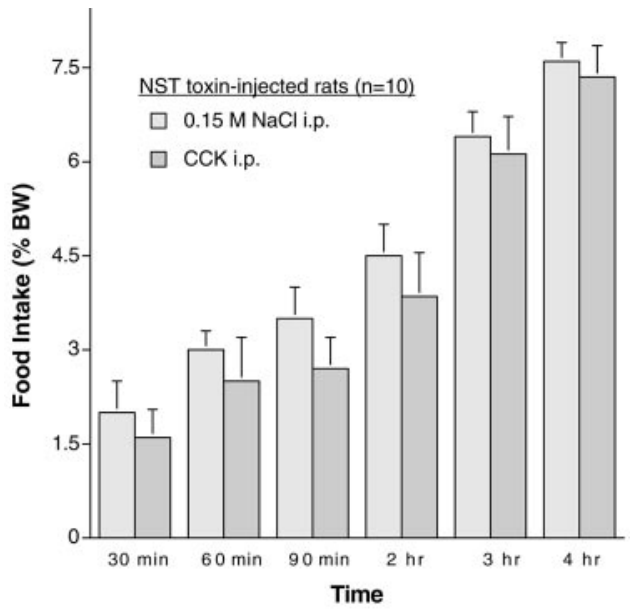

Figure 4. Food intake in rats with previous NST toxin injections $(n=10)$ after control treatment $(0.15 \mathrm{~m} \mathrm{NaCl})$ and after CCK $(10 \mu \mathrm{g} / \mathrm{kg})$. CCK did not significantly inhibit food intake at any time point.

group. The anorexigenic effect of CCK was significant at 30 and $60 \mathrm{~min}$ but not at $90 \mathrm{~min}$ or later (Fig. 3).

The ability of CCK to inhibit food intake was attenuated in rats with bilateral NST toxin injections. Group food intake after CCK treatment was not significantly different from intake after intraperitoneal vehicle at any time point (Fig. 4). However, the effects of CCK treatment were variable within this group. Analysis of data from individual rats revealed that CCK inhibited food intake by $<15 \%$ in six rats with bilateral NST toxin injections but inhibited food intake by $30-60 \%$ in the remaining four (see Fig. 6 ). The ability of CCK to suppress food intake was strongly correlated with NA lesion extent, as described below.

\section{Toxin-induced lesions of DbH-immunopositive neurons}

Bilateral toxin injections were associated with an average $80 \%$ reduction in $\mathrm{DbH}$-positive NST neurons relative to the average number of DbH cells per section in rats with NST vehicle injections (Table 1; Fig. 5, compare $A, B$ ). Loss of DbH-positive NST cells in individual toxin-injected rats ranged from 49.5 to $99.7 \%$ relative to average counts in vehicle-injected rats. These reductions were significant in every case $(p<0.05)$. Thus, toxin injections effectively but variably destroyed $\mathrm{DbH}$-positive NST neurons.

A toxin-related decrement of DbH-positive caudal VLM neurons also was observed in many rats (Table 1). Toxin-injected rats displayed an average of $24 \mathrm{DbH}$-positive VLM neurons per section analyzed, significantly fewer than the average 33 cells per section counted in rats with NST vehicle injections $(p<0.05)$. Thus, in addition to destroying DbH-positive NST neurons, toxin injections often destroyed a subset of DbH-positive VLM neurons.

Qualitative analysis of TH and GLP-1 immunolabeling in three lesioned rats with the most complete loss of $\mathrm{DbH}$-positive NST neurons indicated that the toxin spared dopaminergic neurons in the vagal motor nucleus and caudal NST (Fig. 5C) and GLP-1-positive peptidergic neurons in the caudal NST (Fig. 5D) and adjacent reticular formation. The GLP-1 labeling was further examined quantitatively. The average total number of GLP-1 neurons counted in the three toxin-injected rats (129 \pm 5.7 neurons in five to seven tissue sections per rat) was consistent with a previous report of GLP-1-positive neurons in intact rats (Rinaman, 1999).

\section{Relationship between extent of NST lesion and} CCK-induced anorexia

As reported above, statistical analysis of group data indicated that CCK did not significantly inhibit average food intake in rats with toxin-induced NST lesions (Fig. 4). However, in four rats within this group, CCK suppressed food intake by $\sim 28-60 \%$ relative to intake after intraperitoneal vehicle treatment (Fig. 6). Postmortem analysis of lesion extent indicated that $\sim 33-50 \%$ of $\mathrm{DbH}$ positive NST neurons were spared in these four cases (Table 1, rats $3-5,10)$ relative to average $\mathrm{DbH}$ cell counts in nonlesioned rats. There was a strong positive correlation between the remaining number of NST DbH cells and the magnitude of CCKinduced anorexia at its maximum extent (i.e., at the $30 \mathrm{~min}$ time point) (Fig. 6).

\section{CCK-induced cFos expression in lesioned and nonlesioned rats}

NST cFos activation was significantly greater in lesioned and nonlesioned rats after CCK treatment (i.e., range of 158-263 cells per section; Table 1) compared with intraperitoneal vehicle (i.e., range of 3-39 cells per section; Table 1). On average, somewhat more NST cFos expression was observed after intraperitoneal vehicle treatment in rats with toxin lesions ( $\sim 20$ cells per section) compared with cFos in rats without lesions $(\sim 12$ cells per section), but this difference was not significant. Average NST cFos expression was elevated similarly in lesioned and nonlesioned rats after CCK treatment (i.e., 186 and 205 cells per section, respectively), suggesting that NST toxin lesions eliminated a relatively small subset of the NST neurons activated by CCK (Fig. 5). In a previous report, CCK administered at a dose of $100 \mu \mathrm{g} / \mathrm{kg}$ activated cFos expression in $~ 57 \%$ of GLP-1-positive neurons in intact rats (Rinaman, 1999). The 10-fold lower dose of CCK used in the present study activated cFos expression in a somewhat smaller proportion (i.e., 34.8\% \pm 7.9 ) (Fig. 5D) of GLP-1positive neurons in the three rats with the most extensive loss of DbH-positive neurons.

Relatively few cFos-positive neurons were present in the VLM, lateral PBN, PVN, or CeA in lesioned or nonlesioned rats after intraperitoneal vehicle (Table 1 ). After CCK treatment, $25 \%$ of $\mathrm{DbH}$-positive VLM neurons were activated in nonlesioned rats, whereas a significantly smaller proportion $(11 \%)$ were activated in lesioned rats $(p<0.05)$ (Table 1$)$. VLM activation in lesioned rats after CCK was not significantly different from activation after intraperitoneal vehicle (Table 1). NST lesions were associated with even more marked reductions in CCK-induced cFos expression in the PVN (Fig. 7), including a significant attenuation of cFos expression by OT-positive neurons (Fig. 8). CCK activated an average of 37 OT-positive cells per section analyzed in nonlesioned rats but only approximately six cells per section in lesioned rats (Table 1). These PVN OT activation values were not significantly different in lesioned rats after CCK treatment compared with OT activation after intraperitoneal vehicle (Table 1).

In contrast to the reduced VLM and PVN activation after CCK treatment in lesioned rats, CCK-induced cFos expression within the lateral PBN (Figs. 8, 9) and CeA (Fig. 10) was not attenuated in lesioned rats (Table 1). Despite a clear loss of $\mathrm{DbH}$-positive fibers within the lateral PBN (Fig. 9) and CeA (Fig. 10) in lesioned rats, CCK treatment in lesioned and nonlesioned rats activated statistically similar numbers of CGRP-positive PBN neurons ( $\sim 36$ and $\sim 44$ cells per section, respectively) and similar numbers of cells within the CGRP-rich region of the CeA ( $\sim 218$ and 168 cells per section, respectively) (Table 1 ). 


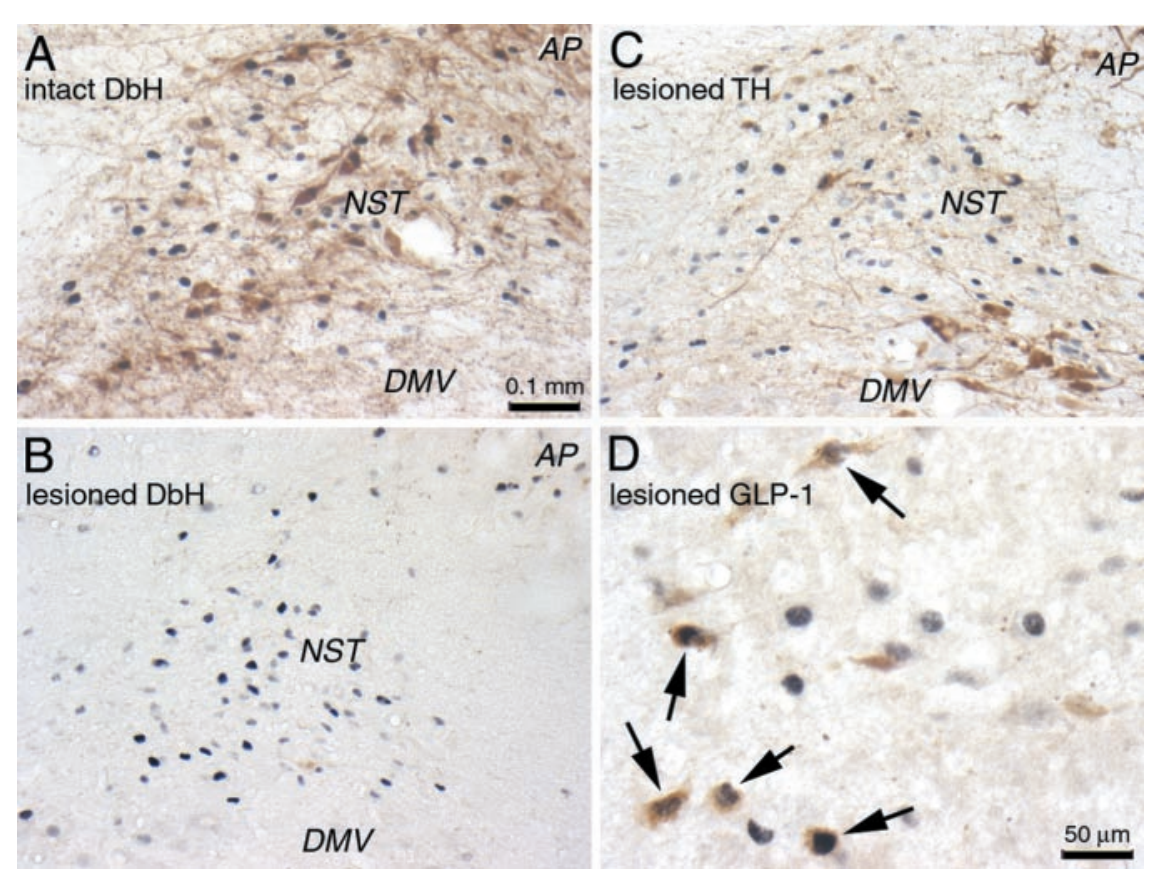

Figure 5. Color photomicrographs depicting CCK-induced cFos expression (blue-black nuclear label) within the dorsal vagal complex in a nonlesioned rat (intact; $A$ ) and in a rat with a toxin-induced lesion of DbH-positive neurons ( $B-D$ are from the same animal). Tissue sections are double labeled for $\operatorname{DbH}(A, B), \mathrm{TH}(C)$, or GLP-1 ( $D$; arrows point out activated neurons). Scale bar in $A$ applies also to $B$ and $C$.

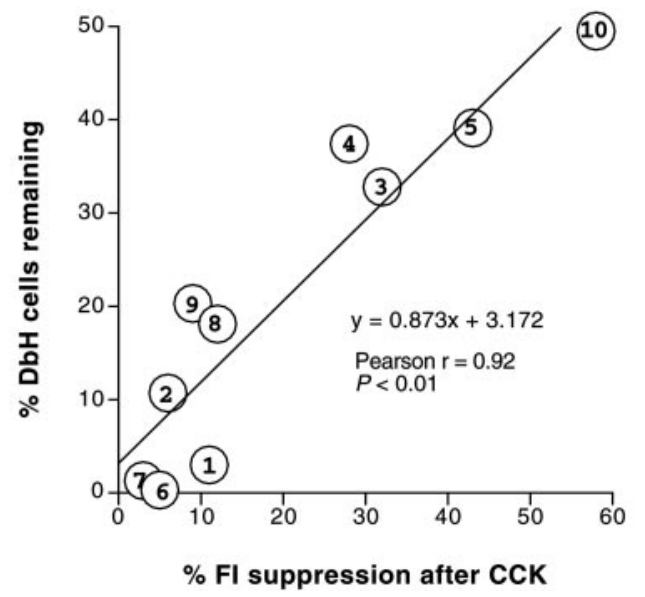

Figure 6. Significant positive correlation between the number of DbH-positive NST neurons remaining in 10 lesioned rats (expressed as percentage of the average number of $\mathrm{DbH}$ cells in nonlesioned rats) and the extent of (CK-induced inhibition of food intake (FI) at the 30 min time point (expressed as percentage of intake in the same rats after intraperitoneal vehicle injection). Circled numbers represent individual rats; see Table 1 for cell counting data.

\section{Discussion}

\section{Saporin toxin lesion efficacy and specificity}

NST microinjections of DbH-saporin toxin conjugate were designed to destroy NA neurons in the caudal medial NST, corresponding to the location of the A2 cell group and the caudal portion of the $\mathrm{C} 2$ cell group. Previous parametric studies attest to the efficacy and specificity of this toxin conjugate (Wrenn et al., 1996; Madden et al., 1999; Ritter et al., 2001; Fraley et al., 2002; Fraley and Ritter, 2003). In the present study, toxin-induced loss of $\mathrm{DbH}$ cell and terminal immunostaining within the caudal DVC and loss of terminal labeling in the PBN, PVN, and CeA offers support for lesion efficacy. Although it is possible that the toxin also destroyed non- $\mathrm{DbH}$-containing neurons, it did not destroy GLP-1-positive neurons, and it also left local TH-positive dopaminergic neurons intact. Furthermore, similar numbers of cFos-positive NST neurons were observed in lesioned and nonlesioned rats after CCK treatment, consistent with evidence that NA neurons comprise a relatively small subset of the total population of NST neurons activated by systemic CCK (Rinaman et al., 1993).

A reduced number of $\mathrm{DbH}$-positive neurons within the VLM was observed in a subset of toxin-injected rats (Table 1 ). Because NA neurons in the VLM are not known to provide synaptic input to the caudal NST, their loss may be attributed to toxin spread from injection sites into the adjacent reticular formation. However, VLM NA cell loss did not occur in every toxin-injected rat and was not necessary for either attenuation of CCK anorexia or for suppression of CCK-induced hypothalamic cFos.

A transient baseline anorexia and BW loss emerged $\sim 7 \mathrm{~d}$ after toxin injections in many rats but gradually resolved when rats had access to liquid diet supplement. Speculatively, dysfunction of NST NA neurons may disrupt digestive vago-vagal reflexes for which local NA signaling mechanisms are implicated (Rogers et al., 2003), thereby leading to gastric malaise and/or early satiety. The gradual recovery of BW gain in lesioned rats suggests that compensatory feeding control systems become operational to restore baseline food intake, despite the loss of NST NA neurons. Indeed, $4 \mathrm{hr}$ food intake was similar in lesioned and control rats after intraperitoneal saline injection. Patterns of ad libitum intake were not analyzed in this study, and it is possible that differences exist between lesioned and control rats in meal number or size.

Correlation between NA cell loss and CCK-induced anorexia A strong positive correlation was found between the number of surviving NA neurons in the caudal NST and the extent of CCKinduced anorexia. As shown in Figure 6, the greater the loss of $\mathrm{DbH}$-positive neurons, the more blunted the ability of CCK to inhibit food intake. A positive correlation between NA neural activation and feeding-induced gastric distension also has been reported (Rinaman et al., 1998). Gastric distension inhibits food intake (Phillips and Powley, 1996), and CCK mimics and amplifies gastric distension vagal afferent signals (Schwartz et al., 1993; Schwartz and Moran, 1998). Thus, not only are NA neurons in the caudal medial NST activated by physiological signals that inhibit food intake, they appear to be necessary for the ability of exogenous CCK to inhibit food intake.

Although the hypothalamus clearly is involved in food intake and energy balance (Watts, 2000; Broberger and Hokfelt, 2001; Berthoud, 2002), it is unclear whether reduced PVN activation in lesioned rats after CCK treatment is associated with the blunted anorexigenic response in those rats. CCK does not activate hypothalamic or other forebrain neurons in neonatal rats, but it inhibits food intake as early as postnatal day 1 (Rinaman et al., 1994). CCK also inhibits sucrose intake in adult decerebrate rats that lack brainstem-hypothalamic connections (Grill and Smith, 1988), and CCK anorexia is preserved in rats after PVN microin- 


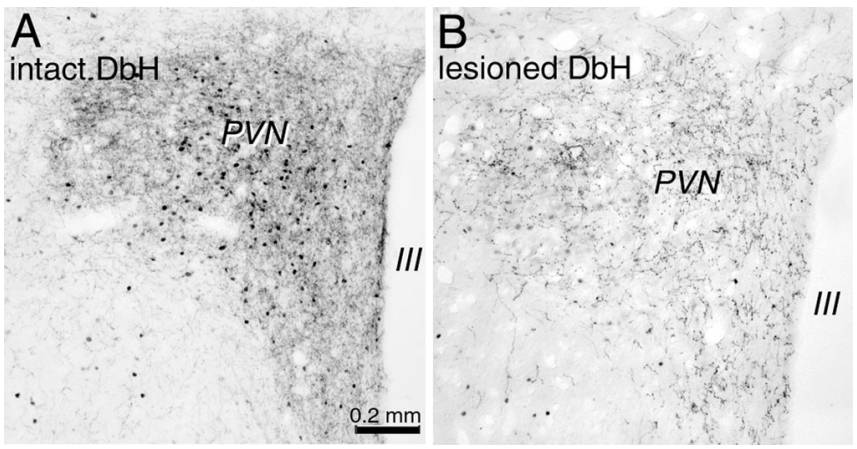

Figure 7. Photomicrographs depicting (CK-induced cFos expression (black nuclear label) within the PVN in a nonlesioned rat (intact; $A$ ) and in a rat with a toxin-induced lesion of $\mathrm{DbH}$-positive neurons (lesioned; $B$ ). Tissue sections are double labeled for $\mathrm{DbH}$; note the reduced PVN CFos expression and DbH fiber labeling in the lesioned rat ( $B$ ). Scale bar in $A$ applies also to B. III, Third ventricle.
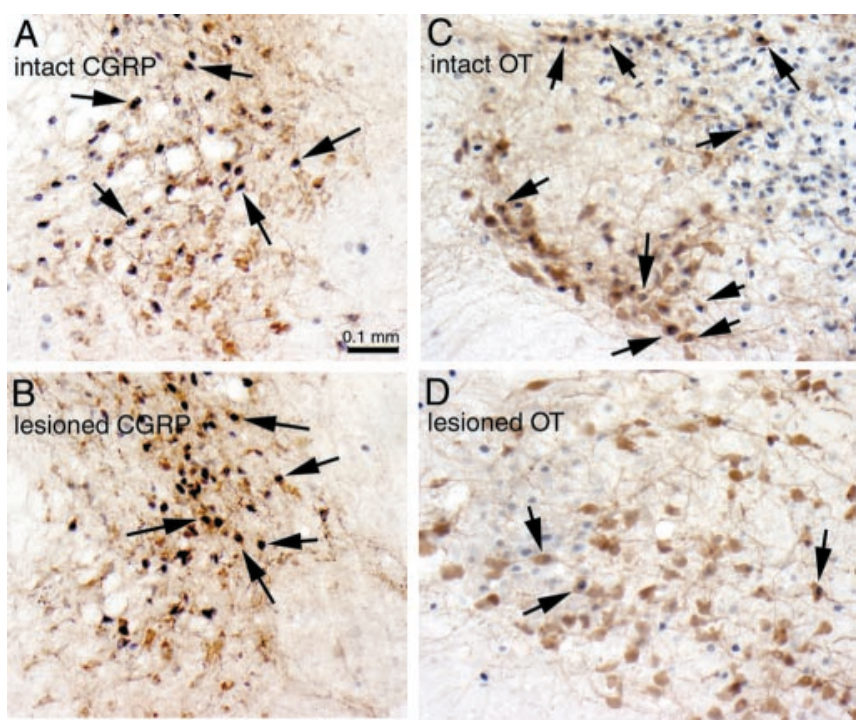

Figure 8. Color photomicrographs depicting CCK-induced cFos expression (blue-black nuclear label) within the lateral PBN $(A, B)$ and PVN $(C, D)$ in a nonlesioned rat ( $A$ and $C$ are from the same rat) and in a rat with a toxin-induced lesion of DbH-positive neurons ( $B$ and $D$ are from the same rat). Tissue sections through the PBN are double labeled for $\operatorname{CGRP}(A, B)$; tissue sections through the PVN are double labeled for $0 T(C, D)$. Arrows point out some of the double-labeled cells visible in each section. Scale bar in $A$ applies to all panels.

jection of saporin toxin to remove its NA innervation (Ritter et al., 2001). In the latter study, $\sim 33 \%$ of NA neurons were left intact in the caudal NST, presumably because they do not project to the PVN toxin injection site. The preserved anorexic response to CCK in that study is consistent with the correlation data obtained in the present study (Fig. 6).

Considered together, these findings support the view that NA neurons in the NST are a necessary and integral part of brainstem circuits that control food intake. These circuits likely include known NA inputs to regions of the medullary and pontine reticular formation (Day et al., 1997) that contain the central pattern generators, premotor, and motor neurons that ultimately control ingestive behavior (Smith, 2000). These integrative and motor circuits also may include a role for NA neurons in the VLM. A previous cFos study reported that CCK activates $\sim 25 \%$ of NA VLM neurons (Rinaman et al., 1993), similar to the present results in nonlesioned rats. Conversely, VLM activation was significantly reduced in lesioned rats after CCK treatment. Because
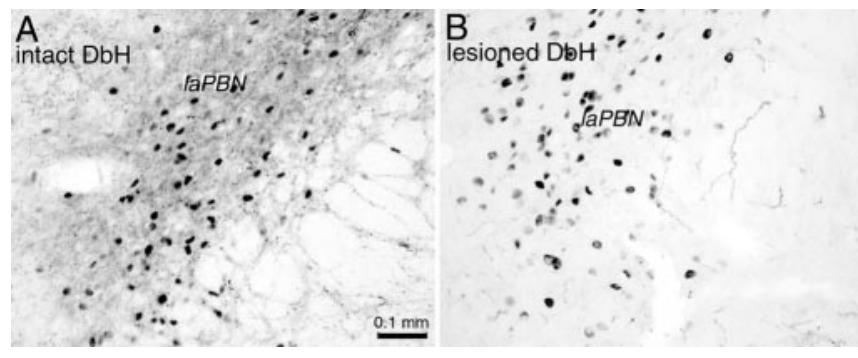

Figure 9. Photomicrographs depicting (CK-induced cFos expression (black nuclear label) within the lateral parabrachial nucleus (laPBN) in a nonlesioned rat (intact; $A$ ) and in a rat with a toxin-induced lesion of DbH-positive neurons (lesioned; $B$ ). Tissue sections are double labeled for $\mathrm{DbH}$; note the reduced $\mathrm{DbH}$ fiber labeling but normally elevated cFos expression in the lesioned rat ( $B)$. Scale bar in $A$ applies also to $B$.

CCK activates $\sim 70 \%$ of VLM neurons that project to the PVN in intact rats (Rinaman et al., 1995), the reduced activation of VLM neurons in lesioned rats also may have contributed to the observed attenuation of PVN cFos expression (discussed further, below).

A recent report indicates that systemic CCK retains its anorexic effects in DbH knock-out mice, in which NA signaling is absent (Cannon and Palmiter, 2003). It will be important to determine whether toxin-induced loss of $\mathrm{DbH}$-containing neurons in mice produces effects similar to those observed in the present study in rats, because there may be a species difference. Alternatively, developmental compensation in $\mathrm{DbH}$ knock-out mice might account for their preserved adult responsiveness to CCK treatment. However, the most likely explanation for the different results obtained in the $\mathrm{DbH}$ knock-out mouse study and the present one is that saporin toxin destroys $\mathrm{DbH}$-positive neurons rather than simply eliminating their NA signaling capacity. NA neurons also release multiple neuropeptides, and concurrent elimination of these signaling molecules from central neural circuits likely contributes to the observed lesion effects.

\section{Dissociation of NA lesion effects on PVN and PBN-CeA activation after CCK treatment}

In addition to inhibiting food intake, systemic CCK increases plasma levels of stress hormones, including ACTH and OT in rats (Verbalis et al., 1986a; Kamilaris et al., 1992) and ACTH and AVP in ferrets, monkeys, and humans (Verbalis et al., 1987; Miaskiewicz et al., 1989; Billig et al., 2001). Exogenous CCK also activates NST NA neurons in ferrets (Billig et al., 2001) and monkeys (Schreihofer et al., 1997), as in rats. Plasma hormone levels were not assayed in the present study, but the blunted PVN cFos expression observed in lesioned rats after CCK treatment predicts a similarly blunted or absent stress hormone response, as reported in neonatal rats (Rinaman et al., 1994).

In contrast to the decremented PVN activation in lesioned rats after CCK, activation in the lateral PBN and CeA apparently was unaffected. The unexpected dissociation between lesion effects on the hypothalamus and amygdala suggests that NA neurons in the caudal NST are unnecessary for conveying gastric viscerosensory signals to the $\mathrm{PBN}$ and $\mathrm{CeA}$. Although the $\mathrm{CeA}$ receives direct inputs from the NST that include a prominent NA component, the $\mathrm{PBN}$ is the main relay for ascending viscerosensory inputs to the CeA (Saper and Loewy, 1980; Milner et al., 1984; Herbert et al., 1990; Jia et al., 1994). CGRP provides a distinct neurochemical marker for the viscerosensory PBN-to-CeA pathway (Yasui et al., 1989), and this pathway appeared to be activated normally in lesioned rats after CCK treatment. Although DbH- 


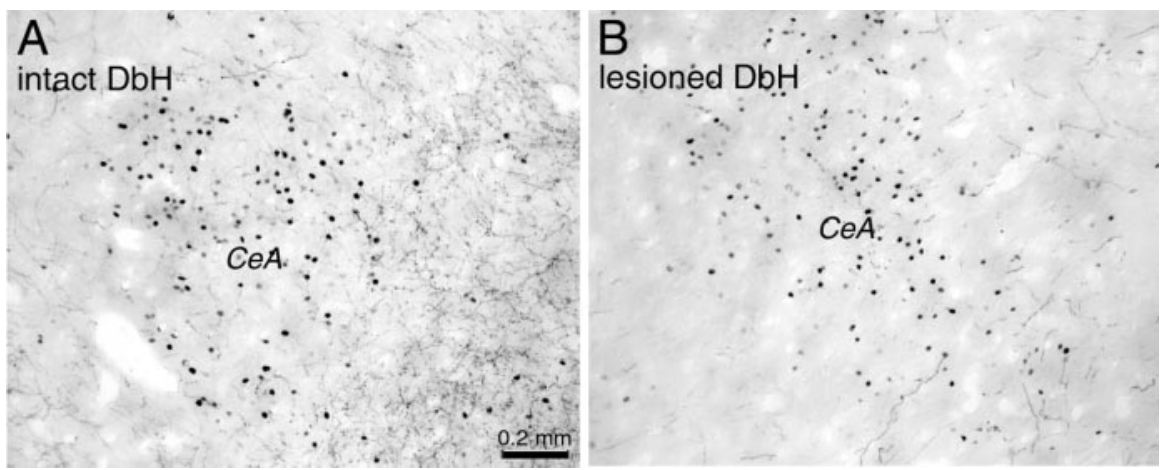

Figure 10. Photomicrographs depicting (CK-induced cFos expression (black nuclear label) within the CeA in a nonlesioned rat (intact; $A$ ) and in a rat with a toxin-induced lesion of DbH-positive neurons (lesioned; $B$ ). Tissue sections are double labeled for DbH; note the reduced DbH fiber labeling but normally elevated cFos expression in the lesioned rat ( $B$ ). Scale bar in $A$ applies also to $B$.
Berthoud H-R (2002) Multiple neural systems controlling food intake and body weight. Neurosci Biobehav Rev 26:393-428.

Billig I, Yates BJ, Rinaman L (2001) Plasma hormone levels and central cFos expression in ferrets after systemic administration of cholecystokinin. Am J Physiol Regul Integr Comp Physiol 281:R1243-R1255.

Broberger C, Hokfelt T (2001) Hypothalamic and vagal neuropeptide circuitries regulating food intake. Physiol Behav 74:669-682.

Cannon CM, Palmiter RD (2003) Peptides that regulate food intake: norepinephrine is not required for reduction of feeding induced by cholecystokinin. Am J Physiol Regul Integr Comp Physiol 284:R1384-R1388.

Curtis KS, Sved AF, Verbalis JG, Stricker EM (1994) Lithium chloride-induced anorexia, but not conditioned taste aversions, in rats with area postrema lesions. Brain Res 663:30-37.

positive NST neurons that project to the PBN and CeA presumably were lesioned, peptidergic NST and AP neurons that project to the PBN and CeA and do not colocalize DbH (cf. Herbert and Saper, 1990) presumably were spared. These include projections arising from GLP-1-positive neurons, which survived the lesions and were activated after CCK treatment. In this regard, a recent report indicates that GLP-1 receptor signaling in the CeA contributes to the formation of conditioned taste aversion (CTA), a sign of nausea, anxiety, and/or visceral illness in rats (Kinzig et al., 2002). It will be interesting to determine whether NA neurons in the NST are necessary or dispensable for CTA formation, because experimental dissociations between the ability of nauseogenic and anxiogenic stimuli to inhibit food intake and produce CTA have been reported previously (Curtis et al., 1994; Kinzig et al., 2002).

\section{Gastric viscerosensory transmission to the hypothalamus and limbic forebrain}

Psychiatrists and gastroenterologists have long appreciated the functional links between gut and brain. For example, the evolving disease model for irritable bowel syndrome (IBS) is a selfperpetuating cycle of positive feedback between the gut and brain, potentially initiated by pathological events in either the CNS or enteric nervous system (Mayer, 1999; Ballenger et al., 2001). Autonomic outputs of the hypothalamus and limbic forebrain are thought to contribute to IBS, which leads to altered viscerosensory feedback from the gut to the brain (Mayer and Raybould, 1990; Ballenger et al., 2001). Anxiety, depression, and panic disorder often are comorbid with IBS and are related to altered neural signaling in the hypothalamus and limbic forebrain (Mayer et al., 2001). It will be important to elucidate the detailed neurochemical and functional organization of neural pathways that convey gastric viscerosensory signals to these forebrain regions. The present results are consistent with the view that hindbrain NA neurons are critical for conveying gastric viscerosensory signals to the hypothalamus but are unnecessary for conveying gastric signals to the amygdala. Additional studies are needed to determine the relative importance of NA and non-NA pathways in mediating the behavioral and physiological effects of viscerosensory stimuli under normal and pathological conditions.

\section{References}

Ballenger JC, Davidson JRT, Lecrubier Y, Nutt DJ, Lydiard RB, Mayer EA (2001) Consensus statement on depression, anxiety, and functional gastrointestinal disorders. J Clin Psychiatry 62 [Suppl 8]:48-51.
Day HE, Campeau S, Watson Jr SJ, Akil H (1997) Distribution of alpha 1a-, alpha $1 \mathrm{~b}$ - and alpha $1 \mathrm{~d}$-adrenergic receptor mRNA in the rat brain and spinal cord. J Chem Neuroanat 13:115-139.

Day HEW, McKnight AT, Poat JA, Hughes J (1994) Evidence that cholecystokinin induces immediate early gene expression in the brainstem, hyporopharmacology 33:719-727.

Edwards GL, Ladenheim EE, Ritter RC (1986) Dorsomedial hindbrain participation in cholecystokinin-induced satiety. Am J Physiol Regul Integr Comp Physiol 251:R971-R977.

Fraley GS, Ritter S (2003) Immunolesion of norepinephrine and epinephrine afferents to medial hypothalamus alters basal and 2-deoxy-Dglucose-induced neuropeptide $Y$ and agouti gene-related protein messenger ribonucleic acid expression in the arcuate nucleus. Endocrinology 144:75-83.

Fraley GS, Dinh TT, Ritter S (2002) Immunotoxic catecholamine lesions attenuate 2DG-induced increase of AGRP mRNA. Peptides 23:1093-1099.

Greenough A, Cole G, Lewis J, Lockton A, Blundell J (1998) Untangling the effects of hunger, anxiety, and nausea on energy intake during intravenous cholecystokinin octapeptide (CCK-8) infusion. Physiol Behav 65:303-310.

Grill HJ, Smith GP (1988) Cholecystokinin decreases sucrose intake in chronic decerebrate rats. Am J Physiol 253:R853-R856.

Herbert H, Saper CB (1990) Cholecystokinin-, galanin-, and corticotropinreleasing factor-like immunoreactive projections from the nucleus of the solitary tract to the parabrachial nucleus in the rat. J Comp Neurol 293:581-598.

Herbert H, Moga MM, Saper CB (1990) Connections of the parabrachial nucleus with the nucleus of the solitary tract and the medullary reticular formation in the rat. J Comp Neurol 293:540-580.

Jia H-G, Rao Z-R, Shi J-W (1994) An indirect projection from the nucleus of the solitary tract to the central nucleus of the amygdala via the parabrachial nucleus in the rat: a light and electron microscopic study. Brain Res 663:181-190.

Kamilaris TC, Johnson EO, Calogero AE, Kalogeras KT, Bernardini R, Chrousos GP, Gold PW (1992) Cholecystokinin-octapeptide stimulates hypothalamic-pituitary-adrenal function in rats: role of corticotropin-

Kinzig KP, D’Alessio DA, Seeley RJ (2002) The diverse roles of specific GLP-1 receptors in the control of food intake and the response to visceral illness. J Neurosci 22:10470-10475.

Madden CJ, Ito S, Rinaman L, Wiley RG, Sved AF (1999) Lesions of the C1 catecholaminergic neurons of the ventrolateral medulla in rats using antiD $\beta$ H saporin. Am J Physiol 277:R1063-R1075.

Mayer EA (1999) Emerging disease model for functional gastrointestinal disorders. Am J Med 107:12S-19S.

Mayer EA, Raybould HE (1990) Role of visceral afferent mechanisms in functional bowel disorders. Gastroenterology 99:1688-1704.

Mayer EA, Craske M, Naliboff BD (2001) Depression, anxiety, and the gastrointestinal system. J Clin Psychiatry 62 [Suppl 8]:28-36.

McCutcheon B, Ballard M, McCaffrey RJ (1992) Intraperitoneally injected thalamus and amygdala of the rat by a $\mathrm{CCK}_{\mathrm{A}}$ receptor mechanism. Neureleasing hormone. Endocrinology 130:1764-1774. 
cholecystokinin-octapeptide activates pica in rats. Physiol Behav 51:543-547.

McLean IW, Nakane PK (1974) Periodate-lysine-paraformldehyde fixative. A new fixative for immunoelectron microscopy. J Histochem Cytochem 22:1077-1083.

Miaskiewicz SL, Stricker EM, Verbalis JG (1989) Neurohypophyseal secretion in response to cholecystokinin but not meal-induced gastric distension in humans. J Clin Endocrinol Metab 68:837-843.

Milner TA, Joh TH, Pickel VM (1984) Substance P, neurotensin, enkephalin, and catecholamine-synthesizing enzymes: light microscopic localizations compared with autoradiographic label in solitary efferents to the rat parabrachial region. J Comp Neurol 226:434-447.

Myers EA, Rinaman L (2002) Viscerosensory activation of noradrenergic inputs to the amygdala in rats. Physiol Behav 77:723-729.

Paxinos G, Watson C (1998) The rat brain in stereotaxic coordinates. Ed 4. New York: Academic.

Phillips RJ, Powley TL (1996) Gastric volume rather than nutrient content inhibits food intake. Am J Physiol 771:R766-R779.

Pudovkina OL, Kawahara Y, de Vries J, Westerink BH (2001) The release of noradrenaline in the locus coeruleus and prefrontal cortex studied with dual-probe microdialysis. Brain Res 906:38-45.

Raybould HE, Gayton RJ, Dockray GJ (1985) CNS effects of circulating CCK8: involvement of brainstem neurones responding to gastric distension. Brain Res 342:187-190.

Rinaman L (1999) Interoceptive stress activates glucagon-like peptide-1 neurons that project to the hypothalamus. Am J Physiol 277:R582-R590.

Rinaman L, Verbalis JG, Stricker EM, Hoffman GE (1993) Distribution and neurochemical phenotypes of caudal medullary neurons activated to express cFos following peripheral administration of cholecystokinin. J Comp Neurol 338:475-490.

Rinaman L, Hoffman GE, Stricker EM, Verbalis JG (1994) Exogenous cholecystokinin activates cFos expression in medullary but not hypothalamic neurons in neonatal rats. Dev Brain Res 77:140-145.

Rinaman L, Hoffman GE, Dohanics J, Le WW, Stricker EM, Verbalis JG (1995) Cholecystokinin activates catecholaminergic neurons in the caudal medulla that innervate the paraventricular nucleus of the hypothalamus in rats. J Comp Neurol 360:246-256.

Rinaman L, Stricker EM, Hoffman GE, Verbalis JG (1997) Central c-fos expression in neonatal and adult rats after subcutaneous injection of hypertonic saline. Neuroscience 79:1165-1175.

Rinaman L, Baker EA, Hoffman GE, Stricker EM, Verbalis JG (1998) Medullary c-Fos activation in rats after ingestion of a satiating meal. Am J Physiol 275:R262-R268.

Ritter S, Bugarith K, Dinh TT (2001) Immunotoxic destruction of distinct catecholamine subgroups produces selective impairment of glucoregulatory responses and neuronal activation. J Comp Neurol 432:197-216.

Rogers RC, Travagli RA, Hermann GE (2003) Noradrenergic neurons in the rat solitary nucleus participate in the esophageal-gastric relaxation reflex. Am J Physiol Regul Integr Comp Physiol 285:R479-R489.
Saper CB, Loewy AD (1980) Efferent connections of the parabrachial nucleus in the rat. Brain Res 197:291-317.

Sawchenko PE, Swanson LW (1981) Central noradrenergic pathways for the integration of hypothalamic neuroendocrine and autonomic responses. Science 214:685-687.

Sawchenko PE, Swanson LW (1982) The organization of noradrenergic pathways from the brainstem to the paraventricular and supraoptic nuclei in the rat. Brain Res Rev 4:275-325.

Sawchenko PE, Li H-Y, Ericsson A (2000) Circuits and mechanisms governing hypothalamic responses to stress: a tale of two paradigms. In: Progress in Brain Research (Mayer EA, Saper CB, eds), pp 61-78: Amsterdam: Elsevier Science.

Schreihofer DA, Cameron JL, Verbalis JG, Rinaman L (1997) Cholecystokinin induces Fos expression in catecholaminergic neurons of the macaque monkey caudal medulla. Brain Res 770:37-44.

Schwartz GJ, Moran TH (1998) Integrative gastrointestinal actions of the brain-gut peptide cholecystokinin in satiety. Prog Psychobiol Physiol Psychol 17:1-34.

Schwartz GJ, McHugh PR, Moran TH (1993) Gastric loads and cholecystokinin synergistically stimulate rat gastric vagal afferents. Am J Physiol 265:R872-R876.

Smith GP (2000) The controls of eating: a shift from nutritional homeostasis to behavioral neuroscience. Nutrition 16:814-820.

Ueta Y, Kannan H, Higuchi T, Negoro H, Yamashita H (1993) CCK-8 excites oxytocin-secreting neurons in the paraventricular nucleus in ratspossible involvement of noradrenergic pathway. Brain Res Bull 32:453-459.

Verbalis JG, McCann MJ, McHale CM, Stricker EM (1986a) Oxytocin secretion in response to cholecystokinin and food: differentiation of nausea from satiety. Science 232:1417-1419.

Verbalis JG, McHale CM, Gardiner TW, Stricker EM (1986b) Oxytocin and vasopressin secretion in response to stimuli producing learned taste aversions in rats. Behav Neurosci 100:466-475.

Verbalis JG, Richardson DW, Stricker EM (1987) Vasopressin release in response to nausea-producing agents and cholecystokinin in monkeys. Am J Physiol 252:R749-R753.

Watson RE, Wiegand ST, Clough RW, Hoffman GE (1986) Use of cryoprotectant to maintain long-term peptide immunoreactivity and tissue morphology. Peptides 7:155-159.

Watts AG (2000) Understanding the neural control of ingestive behaviors: helping to separate cause from effect with dehydration-associated anorexia. Hormones Behav 37:261-283.

Wrenn CC, Picklo MJ, Lappi DA, Robertson D, Wiley RG (1996) Central noradrenergic lesioning using anti-DBH-saporin: anatomical findings. Brain Res 740:175-184.

Yasui Y, Saper CB, Cechetto DF (1989) Calcitonin gene-related peptide immunoreactivity in the visceral sensory cortex, thalamus, and related pathways in the rat. J Comp Neurol 22:487-501. 